\title{
Improving Psychological Well-Being and Quality of Life among Palliative Care Nurses: Literature Review
}

\author{
Manal Baqeas ${ }^{1, *}$, Ahmad Rayan ${ }^{2}$ \\ ${ }^{1}$ MSc /Palliative Care, Graduated from the School of Nursing, the University of Jordan, Amman, Jordan \\ ${ }^{2}$ Assistant professor, Faculty of Nursing, Zarqa University, Zarqa, Jordan \\ *Corresponding author: bakubmh@hotmail.com
}

\begin{abstract}
Background: Nurses who provide palliative care for patients suffering from life threatening conditions often report high levels of psychological distress and poor QOL. Aim: The purpose of this paper was to provide relevant literature regarding the psychological well-being and QOL among palliative care nurses and to suggest specific recommendations to improve psychological health and QOL among nurses provide palliative care. Methods: Literature review of articles related to psychological well-being and QOL among palliative care nurses was searched using CINAHL and PubMed electronic databases using the Keywords "psychological well-being", “ anxiety” ,"stress", "depression", “quality of life”, “nurse”, "palliative care”, and a combination thereof. Results: The current paper highlighted various problems associated with the psychological health and QOL among nurses providing palliative care. Additionally, specific recommendations were provided to improve psychological health and QOL among nurses providing palliative care. Conclusion: All nurses who provide palliative care for patients suffering from life-threatening conditions should be well trained and prepared to provide quality care for patients and to cope effectively with the stressors they might face.
\end{abstract}

Keywords: psychological well-being, quality of life, palliative care, nurse

Cite This Article: Manal Baqeas, and Ahmad Rayan, "Improving Psychological Well-Being and Quality of Life among Palliative Care Nurses.” American Journal of Nursing Research, vol. 6, no. 3 (2018): 82-86. doi: 10.12691/ajnr-6-3-1.

\section{Introduction}

Palliative care is concerned with the management of various and multidimensional symptoms such as pain, grief, depression, difficulty sleeping, fatigue, shortness of breath, constipation, nausea, loss of appetite, and physical complains experienced by patients suffering from life threatening conditions [1]. Palliative care includes the emotional, social, physical, intellectual, and spiritual needs of patients diagnosed with a serious disease. The National Quality Forum (NQF) [2] defined palliative as "patient and family-centered care that optimizes quality of life by anticipating, preventing, and treating suffering." The ultimate goal of palliative care is to improve the quality of life for these patients. There are various terms referring to palliative care such as end-of-life care and hospice care [3]. In the past, the palliative care was directed toward cancer patients [4]. However, the recent approach extending the palliative care to non-cancer patients with life threatening conditions [5].

According to the Worldwide Palliative Care Alliance [6], it was estimated that about 20 million people worldwide require palliative care. Considering the rapid increase in the number of aging population, the prevalence of chronic diseases in the community is expected to dramatically increase and additional patients might need palliative care in the future [7]. Examples of life threatening conditions requiring palliative care are cancer, cardiovascular diseases, HIV/AIDS, chronic pulmonary diseases, kidney failure, liver failure and diabetes [6]. Unfortunately, many patients who need palliative are referred to palliative care lately when the patient is dying [8].

Nurses who provide palliative care for their patients often experience high work-related stress and poor QOL. There are various factors that negatively influence psychological well-being and QOL among palliative care nurses. There is a need to explore these factors and identify specific measures to improve psychological well-being and QOL among these nurses. Improving nurses' psychological health and QOL is essential to help them provide quality care for their patients. Subsequently, the purpose of this paper is to shed the light on psychological well-being and QOL among palliative care nurses. In addition, the current study suggests specific interventions to improve psychological health and QOL among nurses provide palliative care.

\section{Methods}

The authors searched the relevant literature to support their viewpoints with recent evidence.

\section{1, Search Strategy}

Literature review of articles related to psychological well-being and QOL among palliative care nurses was 
searched using CINAHL and PubMed electronic databases using the Keywords "psychological well-being”, "anxiety”, "stress", "depression", “quality of life”, “nurse”, “palliative care”, and a combination thereof.

\subsection{Inclusion Criteria}

Articles of various types of research methods were selected for the review. Literature published in the last five years was prioritized for review.

\subsection{Inclusion Criteria}

Articles that were not written in the English language were excluded from the literature review. In addition, other articles were excluded because they provided redundant data. Further, articles that do not discuss psychological health and QOL among palliative care nurses were excluded from the review.

\section{Results}

\subsection{Psychological Well-Being among Palliative Care Nurses}

Nurses often strive to provide high quality care for patients suffering from life-threatening conditions [9]. In addition, they spend a lot of time at patients' bedsides compared with other health care providers [10]. As a result, they could be substantially influenced by the health care needs of their patients [11]. The literature review suggested that nurses providing palliative care for patients with life threatening condition might report various problems associated with the psychological health. White et al. [12] reported that nurses providing palliative care suffer from feelings of helplessness, frustration, anxiety, depression, distress, and various physical symptoms and complains. Subsequently, providing care for a patient with life-threatening condition or a dying patient is emotionally taxing and difficult for nurses who provide care for this patient. Nurses frequently indicate that providing care for terminally ill and dying patients is frustrating and stressful [13].

The emotional demands and responsibilities of nurses who provide palliative care make them susceptible to increased levels of anxiety and stress [14]. Melvin [15] reported that post-traumatic stress disorder among palliative care nurses could result in burnout among nurses providing palliative care. In addition, previous research studies found high levels stress and anxiety among nurses in addition to other health care professionals providing care for patients receiving end of life palliative care [16]. In addition, nurses providing palliative care might suffer from cumulative emotional and physical effects associated with providing palliative care for the patients over long periods of time [15].

Increasing workload among nurses providing palliative care could be detrimental to nurses' psychological health. Festic et al. [13] argued that high levels of stress experienced by nurses providing end-of-life or palliative care might have negative consequences not only on nurses but also on the health care organization and retention of nurses in their jobs. As a response to high levels of distress, nurses could experience frustration, burnout, stress, and leave their jobs [17]. Because of the high levels of work-related stress and the emotional demands of palliative care nurses, the retention and recruitment of nurses providing hospice care have been a challenge [14]. A study conducted by Festic et al. [13] assessed the levels of distress and its consequences among palliative care nurses and found that about $45 \%$ of the participants have reported a desire to leave their job because of the high levels of distress associated with palliative care. The difficulty of retention and recruitment of palliative care nurses can have a negative impact on the care provided for patients and increase workload among nurses who are currently providing palliative care. Nursing shortage might be additional factor that might influence palliative care nurses. Meier, Lim, and Carlson [18] reported that inadequate number of nursing staff impedes delivery of palliative care and elevate their stress levels. Nurses might report lack of time to meet the demands of patient care and inadequate experience to respond to various needs of patients receiving palliative care [19].

Many nurses who provide palliative care for patients might not have basic education on various topics regarding palliative care. Furthermore, many nurses providing palliative care for their patients are not certified in palliative care. Nurses who provide palliative care for their patients who feel incompetent in this area might suffer from professional compassion fatigue. Subsequently, adequate preparation and training for nurses to provide palliative care might reduce their stress and their professional compassion fatigue.

In addition, misunderstanding regarding the time in which palliative care should be provided could be associated with emotional distress among nurses and other health care professionals. Some nurses and other health care professionals might believe that palliative care is provided only for patients near death, while other nurses believe that it can be provided for patients at any stage of illness [20]. This debate might increase conflicts of nurses with patients, family members, and other health care professionals regarding the patients who have to receive palliative care. Subsequently, there is a need to overcome this barrier to palliative care and accurately assess patients who need to palliative care based on clear policies and guidelines.

Providing care for the patients receiving futile treatment and life-sustaining care such as mechanical ventilators is considered among the most commonly reported stressful aspects of palliative care [21]. In fact, the futile treatment and life-sustaining care might include providing aggressive care that could not result in a survival for patients, which could increase the risk for moral distress among nurses.

Unrelieved symptoms of patients receiving palliative care such as pain, poor self-esteem, nausea and vomiting, fear, and disturbed body image might psychologically affect nurses who frequently providing care for dying patients [22]. Peters et al. [16] found that patient related factors such as working with death and dying patients in addition to workplace and organizational factors are considered stressful situations that frequently reported by palliative care nurses. Working with death and dying 
patients causes high levels of stress, dissatisfaction, grief, and frustration among nurses [23].

\subsection{Quality of Life among Palliative Care Nurses}

The world health organization defined Quality of Life (QOL) as "individuals' perception of their position in life in the context of the culture and value systems in which they live and in relation to their goals, expectations, standards and concerns" [24]. This definition indicates that QOL is a multidimensional and broad concept that includes physical, psychological, social, and environmental aspects of the individual's life. Furthermore, QOL is a complex concept that is influenced by personal cultural beliefs and the personal interaction with the environment.

Literature review suggested that nurses providing palliative care for patients with life threatening condition might report various problems associated with their QOL. Nurses providing palliative care for patients who have difficulty or inability to mobilize their body might have to help these patients to change their position or to do some activities. However, many nurses might frequently move in certain positions such as axial rotation of the trunk in response to an emergent situation related to nursing care provided for the critically ill patients. Overtime, prolong using of this position might cause chronic low back pain and neck pain among nurses which might negatively influence their perception of physical aspects of QOL [25].

As indicated previously, nurses providing end-of-life or palliative care frequently report high levels of anxiety and stress [13]. Various factors such as patients' death, high workload, and relationships with colleagues, patients, and family members could increase anxiety and stress levels in nurses. Previous research suggests that high levels of anxiety and stress might exacerbate physical symptoms and negatively influence nurses' satisfaction with their QOL [26]. In addition, high stress levels directly affect the ability of the individual to maintain healthy interpersonal relationships with others, because the high levels of stress interfere with the affective function and the occupational relationships [27]. Establishing unhealthy interpersonal relationships with others might be associated with poor satisfaction with the social aspects of QOL.

Furthermore, high levels of anxiety and stress might result in poorer psychological well-being [28]. Poor physical and psychological health negatively influences the overall QOL among health care professionals and they are associated with burnout and absence from work [29]. A study was conducted by Machado et al. [30] to examine the association between anxiety, stress, and depression of nurses providing care for patients with life threatening conditions and the levels of attention among these nurses. The results of the study revealed high levels of anxiety, stress, and depression among these nurses. In addition, the high workload among nurses was associated with poor psychological health and impaired attention process in addition to psychomotor decline. Such impairment in cognitive function has an impact on thought, attention, learning, perception, and memory. This is associated with difficulty in carrying out activities of daily living and nursing care to the patients and directly influence quality of life of nurses [31,32]. Subsequently, working with patients who need palliative care might have stressful psychological, physical, and social conditions that influence various aspects of QOL among nurses [33]. Research suggested that, when coping strategies are ineffective, stress might cause severe psychological disorders and eventually reduce the overall quality of life among nurses [34]. Furthermore, Khaghanizadeh et al. [35] indicated that stress negatively affects quality of life of nurses. Quality of life was reported to be poor in all health care professionals employed in hospitals, but impairment in QOL was much more evident among nurses than other health care professionals [36].

Dong, Zhang, Sun, Sang \& Xu [37]. Investigated sleep disturbances among nurses and found that nurses provide care for critically ill patients such as the ICU patients are at high risk for sleep disturbance, which was associated with poor QOL. In addition, occupational stress was found to play a significant role in sleep disturbances among nurses. He et al. [38] assessed the health-related quality of life (QOL) among nurses and compared their scores with scores reported by the general population. The results indicated that nurses had problems with their activity, in addition to pain levels, discomfort, and depression and anxiety, which indicates that nurses have relatively poor QOL in these aspects compared with the general population.

\section{Recommendations for Improving Psychological Well-Being and Quality of Life among Palliative Care Nurses}

Considering the various challenges experienced by palliative care nurses, it is imperative to pay attention to their psychological health and QOL because they may directly affect the quality of nursing care provided for patients. The current paper highlighted clear physical and psychological health consequences for palliative care nurses who provide care for terminally ill patients; therefore, nurse managers should develop specific strategies to support these nurses.

High workload was frequently reported as a modifiable occupational stressor experienced by palliative care nurses. To overcome high workload, health care organizations may want to consider increase staffing and develop specific policies and guidelines to control distractions resulted from family members' interruptions. In addition, there is a need to develop coping skills for palliative care nurses who experience severe emotional responses when providing care for dying patients. Example of these coping skills might include using mindfulness-based interventions $[39,40]$. In addition, Bahmanzadeh \& Alizadeh [41] found that training of nurses to use stress management skills to deal with stress was effective in decreasing stress and anxiety. Jabbari, Hashemi, and Haghayegh [42] showed that the cognitive-behavioral therapy for stress management could improve the psychological well-being and decrease negative thoughts and distortions in thinking among nurses. Additional coping strategies that might protect nurses from the negative consequences of stress include prayer, humor, and social support. 
Considering the busy schedule of palliative care nurses, it is important to highlight that, even using a short-term intervention for stress management could decrease anxiety and stress and increases adaptive coping strategies [43]. Without using adequate coping strategies, stress at workplace could contribute to increase in levels of anxiety and depression, leading to a poor QOL.

Previous research suggests that high levels of anxiety and stress might exacerbate physical symptoms and negatively influence psychological health [26]. Training nurses on relaxation techniques and progressive muscle relaxation might help them to reduce physical tensions and enhance their coping strategies [44]. Furthermore, managing stress among nurses may improve the interpersonal relationships by reducing tension during communications, which might help to establish positive relationships with patients, family members, and colleagues. In fact, social relationships, psychological health, and physical health are considered important components of QOL. Therefore, improving psychological health and interpersonal relationships and decreasing physical exhaustion could substantially improve QOL among palliative care nurses.

As stated previously, many nurses who provide palliative care for patients might not have basic education on various topics regarding palliative care. Furthermore, many nurses providing palliative care for their patients are not certified in palliative care. Lack of knowledge regarding palliative care could contribute to stress and professional compassion fatigue. One important recommendation is to address knowledge deficit regarding palliative care by providing palliative care nurses with educational resources on basic knowledge of palliative care and management of various symptoms reported by patients need palliative care. In addition, all nurses who are intended to provide care for patients suffering from life-threatening conditions should be competent in symptoms management and basic palliative care. Orientation for newly employed nurses should include specific information regarding palliative care at the hospital and its related policies and procedures. Integrating information on palliative care topics into nursing curricula could prepare nursing students to acquire knowledge regarding palliative care before graduation.

\section{Conclusion}

Palliative care nurses were found to experience high work-related stress and poor QOL. Various factors could negatively influence psychological well-being and QOL among palliative care nurses. This paper identified the most commonly reported problems associated with the psychological well-being and QOL among these nurses. All nurses who provide palliative care for patients suffering from life-threatening conditions should be well trained and prepared to provide quality care for patients and cope effectively with the stressors they might face. The current study provided important recommendations to support palliative care nurses and overcome different challenges that they face. Considering the recommendations of the current study, improving nurses' psychological health and QOL is essential to help them provide quality palliative care for patients. However, additional research is needed to identify the most effective methods for improving palliative care nurses' psychological health and QOL.

\section{References}

[1] Quill, T. E., \& Abernethy, A. P. (2013). Generalist plus specialist palliative care-Creating a more sustainable model. The New England Journal of Medicine, 368(13), 1173.

[2] National Quality Forum (2006). A national framework and preferred practices for palliative and hospice care quality (ISBN 1933875-06-2). Washington, DC: National Quality Forum. Retrieved from http://www.qualityforum.org

[3] Wilmont, S. S. (2015). End-of-life care in critical condition. American Journal of Public Health, 105(1), 58-61.

[4] Woo, J., Lo, R., Cheng, J. Y., Wong, F., \& Mak, B. (2011). Quality of end-of-life care for noncancer patients in a non-acute hospital. Journal of Clinical Nursing, 20(13-14), 1834-1841.

[5] Hayle, C., Coventry, P. A., Gomm, S., \& Caress, A. (2013). Understanding the experience of patients with chronic obstructive pulmonary disease who access specialist palliative care: A qualitative study. Palliative Medicine, 27(9), 861-868.

[6] Worldwide Palliative Care Alliance. (2014). Global atlas of palliative care at the end of life. Connor, S., \& Bermedo, M. C., Eds. Retrieved from

http://www.who.int/nmh /Global_Atlas_of_Palliative_Care.pdf

[7] Lau, R., \& O’Connor, M. (2012). Behind the rhetoric: Is palliative care equitably available for all? Contemporary Nurse, 43(1), 56-63.

[8] Cantin, B., Rothuisen, L. E., Buclin, T., Pereira, J., \& Mazzocato, C. (2009). Referrals of cancer versus non-cancer patients to a palliative care consult team: Do they differ? Journal of Palliative Care, 25(2), 92-99. Retrieved from http://search.proquest.com/docview /214200353?accountid=10224

[9] Törnquist, A., Andersson, M., \& Edberg, A. (2013). In search of legitimacy--registered nurses'experience of providing palliative care in a municipal context. Scandinavian Journal of Caring Sciences, 27(3), 651-658.

[10] City of Hope \& American Association of Colleges of Nursing. (2014). ELNEC core Trainthe-Trainer Program 2014. Improving palliative care for patients and families.

[11] Bloomer, M. J., Endacott, R., O’Connor, M., \& Cross, W. (2013). The 'dis-ease' of dying: Challenges in nursing care of the dying in the acute hospital setting. A qualitative observational study. Palliative Medicine, 27(8), 757-764.

[12] White, K., Wilkes, L., Cooper, K., \& Barbato, M. (2004). The impact of unrelieved patientsuffering on palliative care nurses. International Journal of Palliative Nursing, 10(9), 436-444.

[13] Festic, E., Wilson, M. E., Gajic, O., Divertie, G. D., \& Rabatin, J. T. (2012). Perspectives of physicians and nurses regarding end-oflife care in the intensive care unit. Journal of Intensive Care Medicine, 27(1), 45-54.

[14] Harris, L. J. (2013). Caring and coping: Exploring how nurses manage workplace stress. Journal of Hospice \& Palliative Nursing 15(8), 446-454:

[15] Melvin, C. S. (2015). Historical review in understanding burnout, professional compassion fatigue, and secondary traumatic stress disorder from a hospice and palliative nursing perspective. Journal of Hospice and Palliative Nursing, 17(1), 66-72.

[16] Peters, L., Cant, R., Sellick, K., O'Connor, M., Lee, S., \& Burney, S. (2012). Is work stress in palliative care nurses a cause for concern? A literature review. International Journal of Palliative Nursing, 18(11), 561-567.

[17] Kirkey, S. (2014). Doctors and nurses working with critically ill patients suffermoral distress, research finds [Supplemental material]. Postmedia News. Retrieved from http://www.ottawacitizen.com/health/Doctors+nurses+working+w ith+critically+patients/10658168/story.html.

[18] Meier, D. E., Lim, B., \& Carlson, M. D. (2010). Raising the standard: palliative care in nursing homes. Health Affairs, 29(1), 136-140. 
[19] McCourt, R., Power, J. J., \& Glackin, M. (2013). General nurses' experiences of end-of-life care in the acute hospital setting: a literature review. International journal of palliative nursing, 19(10), 510-516.

[20] Perrin, K. O., \& Kazanowski, M. (2015). Overcoming barriers to palliative care consultation.Critical Care Nurse, 36(5), 44-51.

[21] Ferrell, B. R. (2006). Understanding the moral distress of nurses witnessing medically futile care.Oncology Nursing Forum, 33(5), 922-930.

[22] White, K. R., \& Coyne, P. J. (2011). Nurses' perceptions of educational gaps in delivering end of- life care. Oncology Nursing Forum, 38(6), 711-717.

[23] Wallerstedt, B., \& Andershed, B. (2007). Caring for dying patients outside special palliative care settings: experiences from a nursing perspective. Scandinavian Journal of Caring Science, 21, 32-40.

[24] World Health Organization. (1997). Measuring quality of life. Retrieved from http://www.who.int/mental.health/media/68pdf.

[25] Joslin, L., Davis, C., Dolan, P., \& Clark, E. (2014). Quality of life and neck pain in nurses. International journal of occupational medicine and environmental health, 27(2), 236-242.

[26] Toussaint, L., Shields, G. S., Dorn, G., \& Slavich, G. M. (2016). Effects of lifetime stress exposure on mental and physical health in young adulthood: How stress degrades and forgiveness protects health. Journal of health psychology, 21(6), 1004-1014.

[27] Rodrigues, V. M. C. P., \& Ferreira, A. S. D. S. (2011). Stressors in nurses working in Intensive Care Units. Revista latino-americana de enfermagem, 19(4), 1025-1032.

[28] Lovallo, W. R. (2015). Stress and health: Biological and psychological interactions. Sage publications.

[29] Bernal, D., Campos-Serna, J., Tobias, A., Vargas-Prada, S., Benavides, F. G., \& Serra, C. (2015). Work-related psychosocial risk factors and musculoskeletal disorders in hospital nurses and nursing aides: a systematic review and meta-analysis. International journal of nursing studies, 52(2), 635-648.

[30] Machado, D. A., Figueiredo, N. M. A. D., Velasques, L. D. S., Bento, C. A. D. M., Machado, W. C. A., \& Vianna, L. A. M. (2018). Cognitive changes in nurses working in intensive care units. Revista brasileira de enfermagem, 71(1), 73-79.

[31] Faria, C. D. A., Alves, H. V. D., \& Charchat-Fichman, H. (2015). The most frequently used tests for assessing executive functions in aging. Dementia \& Neuropsychologia, 9(2), 149-155.

[32] Kakushi, L. E., \& Évora, Y. D. M. (2014). Direct and indirect nursing care time in an intensive care unit. Revista latinoamericana de enfermagem, 22(1), 150-157.
[33] Moradi, M. F., Ahadi, H., Jomehri, F., \& Rahgozar, M. (2012). Relationship between psychological distress and quality of life in women with breast cancer.'

[34] Circenis, K., Millere, I., \& Deklava, L. (2013). Measuring the professional quality of life among latvian nurses. Procedia-Social and Behavioral Sciences, 84, 1625-1629.

[35] Khaghanizadeh, M., Ebadi, A., \& Rahmani, M. (2008). The study of relationship between job stress and quality of work life of nurses in military hospitals. Journal Mil Med, 10(3), 175-184.

[36] Su, J. A., Weng, H. H., Tsang, H. Y., \& Wu, J. L. (2009). Mental health and quality of life among doctors, nurses and other hospital staff. Stress and Health, 25(5), 423-430.

[37] Dong, H., Zhang, Q., Sun, Z., Sang, F., \& Xu, Y. (2017). Sleep disturbances among Chinese clinical nurses in general hospitals and its influencing factors. BMC psychiatry, 17(1), 241.

[38] He, M., Wang, Q., Zhu, S., Tan, A., He, Q., Chen, T., \& Hu, G. (2012). Health-related quality of life of doctors and nurses in China: findings based on the latest open-access data. Quality of Life Research, 21(10), 1727-1730.

[39] Rayan, A. H. R. (2017). Mindfulness, Quality of Life, and Severity of Depressive Symptoms Among Patients With Schizophrenia and Patients With Major Depressive Disorder. Journal of psychosocial nursing and mental health services, 55(5), 40-50.

[40] Rayan, A., \& Ahmad, M. (2018). Psychological Distress in Jordanian Parents of Children With Autism Spectrum Disorder: The Role of Trait Mindfulness. Perspectives in psychiatric care, 54(1), 11-18.

[41] Bahmanzadeh, L \& Alizadeh, K. (2017). The effect of cognitivebehavioral stress management training on improving psychological symptoms and quality of life in nurses. Journal of Research \& Health Social Development \& Health Promotion Research Center, 7(6), 1154-1161

[42] Jabbari, Z., Hashemi, H., \& Haghayegh, S. A. (2012). Survey on effectiveness of cognitive behavioral stress management on the stress, anxiety, and depression of pregnant women. 8 (7), 1341 To 1347.

[43] Rayan, A., \& Ahmad, M. (2017). Effectiveness of mindfulnessbased intervention on perceived stress, anxiety, and depression among parents of children with autism spectrum disorder. Mindfulness, 8(3), 677-690.

[44] Prato, C. A., \& Yucha, C. B. (2013). Biofeedback-assisted relaxation training to decrease test anxiety in nursing students. Nursing education perspectives, 34(2), 76-81. 